\title{
Stability issues in bioanalysis
}

\author{
Chad Briscoe ${ }^{*, 1}$ \& Rafiq Islam ${ }^{2}$ \\ ${ }^{1}$ PRA Health Sciences, Lenexa, KS 66221, USA \\ ${ }^{2}$ Celerion, Lincoln, NE 68502, USA \\ *Author for correspondence: BriscoeChad@prahs.com
}

First draft submitted: 24 September 2019; Accepted for publication: 15 October 2019; Published online: 2 December 2019

Keywords: bioanalysis • stability

Stability, or lack there-of, is a fundamental principle to identify, assess and if possible mitigate failures in the field of bioanalysis. On the surface, stability assessment is straightforward. What is the concentration when preparing the test samples and what is the concentration following a specified storage period at a specified temperature. However, any bioanalytical scientist working in a regulated environment knows there is much more to it than that. We must differentiate stability from what appears to be stability, we must determine where and how stability issues are affecting the measurements, and we must figure out how the new drug constructs and measurement approaches change the paradigm of what we knew before. The approaches used to conduct stability experiments (i.e., whole blood stability, number of tubes or definition of fresh sample measurement) also varies significantly among laboratories. We are also experiencing significant scrutiny from regulatory agencies on how stability experiments are conducted. It is because of these ever emerging challenges in regulated bioanalysis that we decided it was appropriate to revisit the topic of stability in a focused issue in the journal at this time. It is a very exciting time to be in the pharmaceutical industry because of the explosion of new modalities and new measurement approaches, which also adds complexity on how stability experiments are conducted and the how the data in interpreted. In this issue, we have covered many of the new and some of the old challenges with a fresh new light. We hope that you will find this to be an interesting and useful reference to solve your challenges in the various areas that we have garnered submissions.

We are excited to have enlisted several authors to take a fresh look at some older but still fundamental topics in stability and we applaud the authors for taking the time to present a new angle for us. Ohnmacht et al. have collected a number of case studies on sample collection and handling [1]. A challenge that is critical to success in clinical trial execution. On a similar theme, Anderson's Special Report illustrates the importance of ensuring the biological sample integrity throughout the entire process from collection to analysis including challenges associated with rare matrices, microsampling dried blood spot storage issue and analytes with know instability [2]. MacNeil has taken on an important and sometimes overlooked challenge in stability, which is the day-to-day reality of utilizing solvents, reagents and additives [3]. The reagents used in routine laboratory experiments can have significant impact on the stability of test samples. Finally, Cape and her colleagues discussed the current approaches, challenges and regulatory aspects around the measurement of stability in whole blood [4].

Another area of stability measurement that has been evolving in recent years is in regard to regulatory expectations around testing of stability. The testing approach and types of testing required changes as regulators and industry scientists identify new issues. No topics have been more controversial in the industry than coadministered compound stability, assessment of multiple tubes for stability tests and stability of antidrug antibodies. We greatly appreciate Haidar et al.'s contribution to the topic of three tube stability requirements in which they present background and results of the US FDA's internal lab testing results for three tubes versus one tube stability [5]. I think you will find the outcome very interesting as we did. We are likewise grateful for the contribution of Zimmer and her colleagues for their work on coadministered compound stability in which they take on a controversial topic in an informative and well-researched approach [6]. Finally, the team lead by Boridy and Xing provided an excellent and thoughtful piece on the stability measurements, particularly for long-term stability, of antidrug antibodies [7].

It was also our goal to present stability issues related to the new drug constructs and technologies for measurement. Therefore, we are pleased to have three excellent contributions in this area. Kakkanaiah et al. presented their work related to cellular pharmacokinetic $(\mathrm{PK})$ and pharmacodynamic (PD) assessments with flow cytometry [8]. An 
important contribution as we see the rise in cellular therapies and flow cytometry-related analysis in general. On a similar theme, Muruganandham et al. have addressed their approach to measurement of stability in regulated flow cytometry [9]. A critical topic as we see more and more flow cytometry-based analysis being included to support regulatory submissions for novel therapeutics. Lastly, the inclusion of biomarkers has become routine in most clinical studies. Therefore, the approach and expectations for how to validate the assays has also gained more scrutiny. There are special challenges to the measurement of the stability in biomarkers, and Islam and colleagues have done a nice job to tackle many of these key challenges in their paper on the topic [10].

We are grateful for the contribution of all of our authors and they have our sincere thanks for their tremendous efforts. We have learned a lot ourselves from the opportunity to review these papers and we think you will also.

\section{Financial \& competing interests disclosure}

The authors have no relevant affiliations or financial involvement with any organization or entity with a financial interest in or financial conflict with the subject matter or materials discussed in the manuscript. This includes employment, consultancies, honoraria, stock ownership or options, expert testimony, grants or patents received or pending, or royalties.

No writing assistance was utilized in the production of this manuscript.

\section{References}

1. Ohnmacht C, Cooley M, Darling R, Lei S, Patel V. Sample stabilization strategies: a case study review of unique sample collection and handling procedures. Bioanalysis 11(20), 1867-1880 (2019).

2. Anderson M. Ensuring biological sample integrity from collection to analysis for LC-MS workflows: case studies illustrating challenges in clinical trials. Bioanalysis 11(20), 1859-1866 (2019).

3. MacNeil R. Stability considerations associated with solvents, solutions and additives in quantitative chromatographic methods. Bioanalysis 11(20), 1815-1817 (2019).

4. Ledvina AR, Ewles M, Pang Y, Cape S. Whole blood stability in quantitative bioanalysis. Bioanalysis 11(20), 1885-1897 (2019).

5. Haidar SH, Shakleya D, Wang J, Cai X, Faustino P. Evaluation of stability using one versus three tubes for each quality control concentration in matrix-based bioanalysis. Bioanalysis 11(20), 1823-1835 (2019).

6. DeChenne S, Yahvah K, Zimmer J. Validating stability and selectivity in the presence of co-administered compounds. Bioanalysis 11(20), 1819-1821 (2019).

7. Boridy S, Xing K. Assessing the long-term stability of anti-drug antibodies in method validation: what is the added value? Bioanalysis 11(20), 1899-1903 (2019).

8. Kakkanaiah VN, Pan F, Bennett P. Evaluation of sample stability for cellular kinetics and a pharmacodynamic flow cytometry methods. Bioanalysis 11(20), 1881-1884 (2019).

9. Muruganandham A, Dumont C, Xing K. Approaching stability challenges for flow cytometry in a regulated bioanalytical environment. Bioanalysis 11(20), 1845-1858 (2019).

10. Kar S, Islam C. New approaches for biomarker stability determination in regulated bioanalysis: trending, bridging, and incurred samples. Bioanalysis 11(20), 1837-1844 (2019). 